Original Research Paper

\title{
The Hypoxia-Inducible Factor-1 $\alpha$ Signaling Pathway and its Relation to Cancer and Immunology
}

\author{
${ }^{1,2}$ Bruno R.B. Pires, ${ }^{3}$ Andre L. Mencalha, ${ }^{1,2}$ Gerson M. Ferreira, ${ }^{4}$ Carolina Panis, ${ }^{1,2}$ Rafael C.M.C. Silva and \\ ${ }^{1,2}$ Eliana Abdelhay
}

${ }^{1}$ Laboratório de Célula-Tronco, Instituto Nacional de Câncer José Alencar Gomes da Silva, Rio de Janeiro, RJ, Brazil ${ }^{2}$ Instituto Nacional de Ciência e Tecnologia para o Controle do Câncer, Brazil

${ }^{3}$ Departamento de Biofisica e Biometria, Universidade do Estado do Rio de Janeiro, Rio de Janeiro, RJ, Brazil

${ }^{4}$ Centro de Ciências Médicas, Universidade Estadual do Oeste do Paraná, Unioeste, Brazil

Article history

Received: 15-01-2014

Revised: 24-07-2014

Accepted: 05-01-2015

Corresponding Author:

Bruno R.B. Pires

Laboratório de Célula-Tronco, Instituto Nacional de Câncer José Alencar Gomes da Silva, Rio de Janeiro, RJ, Brazil

Email: carolpanis@sercomtel.com.br

\begin{abstract}
Hypoxia-Inducible Factor 1 (HIF-1) is a transcription factor that is present in all metazoans from early embryonic development throughout adult life. It plays a major roles in the different stress responses that are triggered by low Oxygen $\left(\mathrm{O}_{2}\right)$ levels and its expression is associated with cell survival. HIF-1 is a heterodimer protein that comprises the subunits HIF- $1 \alpha$ and HIF-1 $\beta$. The HIF- $1 \alpha$ subunit is regulated by $\mathrm{O}_{2}$-dependent hydroxylation of proline and asparagine residues, which results in ubiquitination and subsequent proteasome degradation. It may also be regulated independently of $\mathrm{O}_{2}$. This review discusses the regulatory mechanisms and biological significance of HIF-1 $\alpha$ with regard to cancer development and immune regulation. HIF- $1 \alpha$ stabilization under hypoxic conditions is crucial to the survival of established tumors and cancer stem cells. HIF- $1 \alpha$ is included in the hallmarks of cancer that are related to energy metabolism, although there is clear evidence that this transcription factor might participate in other hallmarks, such as angiogenesis, invasion and metastasis, self-sufficiency in proliferation signals and even apoptosis evasion. With regard to immunology, HIF-1 $\alpha$ regulates Interleukin (IL)-1 $\beta$, IL-8 and Heme-Oxygenase-1 (HO-1) and despite some conflicting results, HIF- $1 \alpha$ is considered to be an important component of innate immune cellmediated inflammation. With regard to the adaptive immune response, HIF-1 $\alpha$ expression is related to Th17 polarization and Treg inhibition. Thus, the HIF-1 $\alpha$ signaling pathway has been designated as a promising target for new drugs in several studies.
\end{abstract}

Keywords: Hypoxia-Inducible Factor 1, HIF-1 $\alpha$, Hypoxia, Cancer, Immunology

\section{Introduction}

Oxygen $\left(\mathrm{O}_{2}\right)$ is essential to the physiology and homeostasis of animal cells. When cells are exposed to low $\mathrm{O}_{2}$ levels (hypoxia), they express and stabilize the Hypoxia-Inducible Factor 1 (HIF-1) protein, blocking its degradation. HIF-1 plays a major role in different stress responses that are triggered by low $\mathrm{O}_{2}$ levels and its expression is associated with cell survival (Wang et al., 1995). HIF-1 is present in all metazoans from early embryonic development throughout adult life. The HIF1 protein belongs to the family of transcription factors with a basic Helix-Loop-Helix domain (bHLH) coupled to a PAS (PER-ARNT-SIM) domain, which provides more stability to dimerizations that involve HIF-1 (Wang et al., 1995).

\section{The Hypoxia-Inducible Factor-1 Signaling Pathway and its Subunits}

HIF-1 is a heterodimer protein that comprises the subunits HIF- $1 \alpha$ and HIF- $1 \beta$. It binds to DNA at a consensus sequence: 5'-[A/G]CGTG-3'; there may also be a second consensus sequence 5'-[A/C]ACAG-3' near the classical one (Semenza, 1998; 2007). Under normal oxygen conditions (normoxia), HIF- $1 \alpha$ is continuously synthesized and degraded and its regulation directly depends on $\mathrm{O}_{2}$ levels (Fig. 1). Under hypoxic conditions, 
the proteins that block interaction with HIF-1 $\alpha$ are degraded, favoring dimerization with HIF-1 $\beta$, which is constitutively expressed. Thus, HIF- $1 \alpha$ is stabilized, recruits coactivators and becomes structurally capable of regulating hundreds of genes in an extremely refined way (Fig. 1) (Semenza, 2007).

The HIF-1 $\alpha$ subunit is regulated by $\mathrm{O}_{2}$-dependent hydroxylation of proline and asparagine residues, which results in ubiquitination and subsequent proteasome degradation. These hydroxylation reactions are responsible for the activation of a mechanism that responds to $\mathrm{O}_{2}$ levels. This mechanism is then transduced to the cell nucleus and promotes the activation of specific genes through HIF-1 activity (Semenza, 1998; 2007).

The hydroxylation of proline residues occurs via the Prolyl Hydroxylase Domain protein 2 (PHD2) on Pro ${ }^{402}$ or Pro ${ }^{564}$, or both. This hydroxylation promotes the binding of the Von Hippel-Lindau (VHL) protein, which interacts with Cullin-2, Elongin $\mathrm{B}$ and $\mathrm{C}$ proteins, blocking transcriptional elongation activity and recruiting the ubiquitin-protein ligase E3 complex and ultimately resulting in the ubiquitination and subsequent degradation of HIF- $1 \alpha$ via the $26 \mathrm{~S}$ proteasome (Fig. 1). PHD2 also converts $\mathrm{O}_{2}$ and $\alpha$-ketoglutarate, generating succinate and $\mathrm{CO}_{2}$ as products. The enzymatic activity of PHD2 is reduced in hypoxia due to a decrease in substrate availability. This occurs by direct inhibition of the catalytic center containing Fe (II) and/or by Reactive Oxygen Species (ROS) produced via the mitochondrial

Normoxia

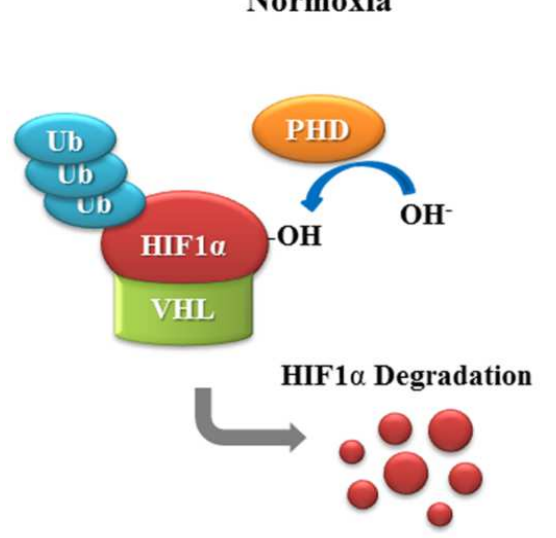

$\mathrm{O}_{2}$ Levels respiratory chain. Although only PHD2 is described in this process, the three isoforms (PHD1, PHD2 and PHD3) also promote HIF-1 $\alpha$ degradation. The hydroxylation of asparagine is catalyzed by Factor Inhibiting HIF-1 (FIH-1) on $\mathrm{Asn}^{803}$, which blocks the binding of transcriptional co-activating proteins p300 (300-kilodalton coactivator protein) and CBP (CREBbinding protein), consequently reducing the transcription of the HIF- $1 \alpha$ target genes (Semenza, 2007).

The half-life of HIF-1 $\alpha$ may also be regulated independently of $\mathrm{O}_{2}$, whereby the Receptor for Activated C Kinase 1 (RACK1) competes for the binding site of the chaperone Heat Shock Protein 90 (HSP90), which is involved in the protein stabilization of HIF-1 $\alpha$ and several growth factors (Sawai et al., 2008). Thus, RACK 1 protein interacts directly with Elongin C, routing HIF-1 $\alpha$ for ubiquitination and proteasomal degradation independently of PHD2 or VHL (Benezra et al., 1990). It is important to emphasize that HIF- $1 \alpha$ is not completely degraded after it is ubiquitinated, due to the ubiquitin removal by VHL Deubiquitinating Enzyme 2 (VDU2), which stabilizes the HIF-1 $\alpha$. Recently, Foxler et al. (2012) demonstrated the importance of the tumor suppressor LIM DomainContaining Protein (LIMD1) in regulating the levels of HIF-1 $\alpha$. LIMD1 acts as a scaffold for PHDs and VHL, thereby forming the PHD/LIMD1/VHL complex. The loss of function of LIMD1 triggers an increase in HIF$1 \alpha$ levels under both hypoxia and normoxia conditions.

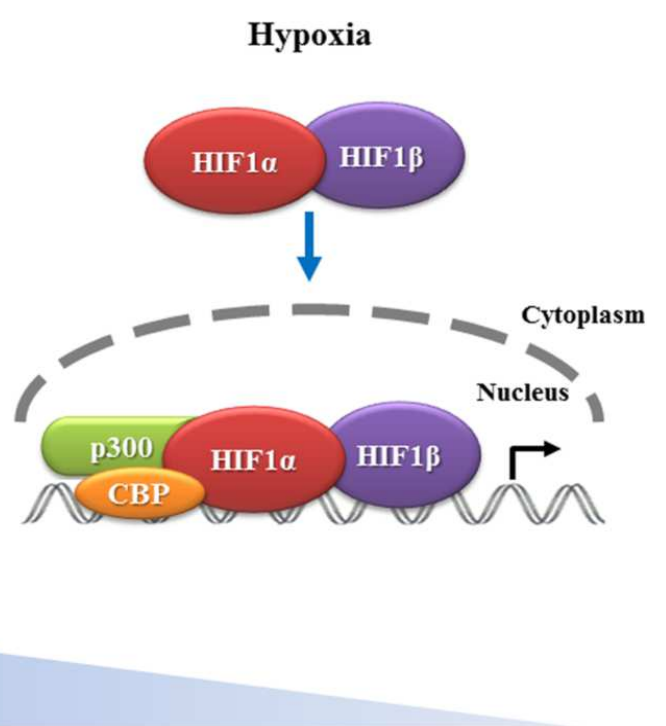

Fig. 1. Under normal oxygen conditions (normoxia), HIF-1 $\alpha$ is continuously synthesized and degraded. The HIF-1 $\alpha$ subunit is regulated by O2-dependent hydroxylation reactions catalyzed by Prolyl Hydroxylase Domain Protein (PHD), that promotes the binding of the Von Hippel-Lindau (VHL) protein, resulting in the ubiquitination and subsequent degradation of HIF-1 $\alpha$ via proteasome. Under hypoxic conditions, the proteins that block interaction with HIF-1 $\alpha$ are degraded, favoring dimerization with HIF-1 $\beta$, which is constitutively expressed. Thus, HIF-1 $\alpha$ is stabilized, translocates into the nucleus, recruits the transcriptional co-activating proteins p300 (300-kilodalton coactivator protein) and CREB-Binding Protein (CBP) and becomes structurally capable of binding to DNA at a consensus sequence and activates the target genes 


\section{Hypoxia-Inducible Factor-1 $\alpha$ in Physiological Processes and Cancer}

The HIF family genes are the major transcriptional factors involved in cell survival under low oxygen conditions. This ability allowed mankind to conquer physiologically challenging environments, such as the high altitudes of the Himalayas and the Andes where oxygen availability is diminished. Yi et al. (2010) sequenced the genome of 50 Tibetans who live in the Himalayas in order to find evidence of a genetic component in their ability to adapt to high altitudes. The results showed that the EPAS1 gene in the highaltitude population had $78 \%$ more Single-Nucleotide Polymorphisms (SNPs) than the gene in the lowaltitude population. This gene encodes the protein HIF- $2 \alpha$ and its polymorphism is strongly associated with protein stabilization and increased activity in different processes such as erythropoiesis. The HIF- $2 \alpha$ protein shares a $48 \%$ similarity with HIF- $1 \alpha$ and is also regulated by PHD2. HIF- $2 \alpha$ is also able to dimerize with HIF-1 $\beta$ and bind to the same target sequence as the HIF- $1 \alpha / \mathrm{HIF}-1 \beta$ heterodimer. Although there are many similarities, HIF- $1 \alpha$ and HIF- $2 \alpha$ must be considered as two separate entities because they perform different functions depending on where they are expressed in the tissue (Semenza, 2007). Because HIF- $1 \alpha$ has a greater association with cancer than HIF$2 \alpha$, this particular review focuses on HIF-1 $\alpha$.

During embryonic development, HIF-1 $\alpha$ is responsible for stimulating the expression of many genes: Octamer-Binding Transcription Factor 4 (OCT4), which is essential for the maintenance of a pluripotent phenotype in embryonic stem cells (Nichols et al., 1998); the inducer Vascular Endothelial Growth Factor (VEGF) (Ferrara et al., 2003); the cytokine Erythropoietin (EPO) (Krantz, 1991); the embryonic growth factor Insulin-like Growth Factor 2 (IGF2) (Baker et al., 1993); Inhibitor of DNA binding 2 (ID2), an inhibitor of the HLH binding domain of DNA (Benezra et al., 1990) and NOTCH1, which plays a role in development by controlling cell fate (ArtavanisTsakonas et al., 1999). Mazumdar et al. (2010) proposed a close association between HIF- $1 \alpha$ and $\mathrm{WNT} / \beta$-catenin signaling, which results in embryonic, neural and hematopoietic stem cell maintenance, including selfrenewal and proliferation. Interestingly, the deletion of HIF- $1 \alpha$ in mouse embryonic stem cells leads to the absence of $L E F 1$ and $T C F$ expression, the effectors of WNT signaling. In addition, experiments confirmed the direct binding of HIF-1 $\alpha$ onto the LEF1 and TCF promoter regions under hypoxic conditions. It is not completely clear how stem cell niche is regulated by hypoxia, but because research has shown that stem cells under lower oxygen conditions are less exposed to ROS and consequently have a lower risk of mutagenesis (Kaufman, 2010; Adikwu and Brambaifa, 2012; Hazra et al., 2012), a protective effect on the genome may be considered. The WNT/ $\beta$-catenin signaling pathway has been extensively studied in cancer research and is related to metastatic processes and resistance to therapies. Likewise, HIF-1 $\alpha$ plays an important function via $\mathrm{WNT} / \beta$-catenin activation, affecting tumor development and stem cell maintenance during cancer and death resistance (Kaufman, 2010).

In cancer, the loss of tumor suppressor VHL function is often observed. This loss triggers greater stability and accumulation of HIF- $1 \alpha$ and HIF-2 $\alpha$, consequently enabling their role in carcinogenesis (Kaelin, 2008). Moreover, previous research has shown that stabilization of HIF-1 $\alpha$ under hypoxic conditions is crucial to the survival of both established tumors and cancer stem cells (Semenza, 2010).

HIF-1 $\alpha$ also orchestrates the Warburg Effect, an alternative metabolic process of cancer cells, in which more oxygenated cells transfer glucose to hypoxic cells that overexpress Glucose Transporter (GLUT1). Under hypoxia, glucose is anaerobically converted into lactate to generate ATP. The lactate is exported to the aerobic cells by overexpression of Monocarboxylate Trasporter (MCT1). This lactate is converted into pyruvate by $L D H$ (Lactate Dehydrogenase enzyme), which is expressed at a higher level in normoxia cells (Cairns et al., 2011). Thus, a feedback system is created whereby energy sources are shuttled between metabolic cells that are located in different microenvironments. Interestingly, this entire process occurs in response to environmental conditions and is carried out by a gene expression pattern that is transcriptionally regulated by HIF-1 $\alpha$. The effect that this transcription factor has on energy metabolism was described by Gregg Semenza, who showed that HIF$1 \alpha$ acts as a regulator of glycolytic enzymes under low oxygen conditions and is overexpressed in the hypoxic regions of tumors (Denko, 2008).

The constitutive expression of the HIF-1 complex is frequently associated with tumor progression, poor survival and even resistance to therapies. In this context, the mechanism that implicates HIF-1 in radioresistance has been recently revised by Meijer et al. (2012). After radiotherapy, the hypoxic tumor cells that survive radiation are forced to re-oxygenate because the surrounding cancerous population has been killed. Therefore, a wide array of HIF-1-related events is triggered by this re-oxygenation process inside the tumor. A primary acute consequence of tumor irradiation is the downregulation of HIF-1 activity; however, the long-term accumulation of ROS re-stabilizes the HIF-1 molecule and results in increasing levels of HIF-1 in the hours soon after radiotherapy, which may last over a week. This 
upregulation of HIF-1 triggers other pro-angiogenic cascades, such as VEGF, which protects the microvasculature of the tumoral mass against radiation-induced apoptosis. A secondary mechanism induced by HIF-1 involves enhancing the antioxidant capacity of cells by starting their glycolytic metabolism, which makes the tumor resistant to virtually any type of oxidative stress-driven chemotherapy (Meijer et al., 2012). Regardless of the specific mechanism, augmented HIF-1 expression is strongly correlated with poor disease prognosis and death.

\section{Hypoxia-Inducible Factor-1 $\alpha$ as a Hallmark of Cancer}

Hanahan and Weinberg (2011) highlighted HIF-1 $\alpha$ in the group of cancer hallmarks that are related to energy metabolism, although there is clear evidence that HIF-1 $\alpha$ may participate in other hallmarks, such as angiogenesis, invasion and metastasis, selfsufficiency in proliferation signals and even apoptosis evasion. In response to hypoxia, HIF-1 $\alpha$ regulates the $V E G F$ gene family during angiogenesis. This regulation occurs in a physiological manner that is independent of oxygen levels. Regarding invasion and metastasis, HIF-1 $\alpha$ has been positively correlated with other transcription factors, such as TWIST1, which is one of the master regulators of epithelialmesenchymal transition and is responsible for the phenotypic alteration of cancer cells during metastasis (Yang et al., 2008). Self-sufficiency in proliferation signals was demonstrated in a recent study by Wang et al. (2012). The study showed that HIF signaling involves CAV1 (Caveolin 1), the main component of caveolae in the plasma membrane (Okamoto et al., 1998), culminating in the activation of Epidermal Growth Factor Receptor (EGFR), the cell surface receptor of the epidermal growth factor family and ExtracellularSignal-Regulated Kinases (ERK), which is also known as Mitogen-Activated Protein (MAP) Kinase that triggers several signaling pathways (Boulton and Cobb, 1991) leading to exacerbated cell proliferation. Zhang et al. (2008) investigated apoptosis evasion and found that mitochondrial autophagy is dependent on HIF-1 $\alpha$, which aids cell survival in the absence of nutrients and decreases the generation of mitochondrial ROS. In addition, mitochondrial autophagy is consistently found in cancer, especially in environments of intratumoral hypoxia, inducing a "silent" death of the mitochondria without releasing cytochrome c, which triggers intrinsic apoptosis (Zhang et al., 2008). A schematic picture about the biological processes and signalings regulated by HIF- $1 \alpha$ is shown in Fig. 2.

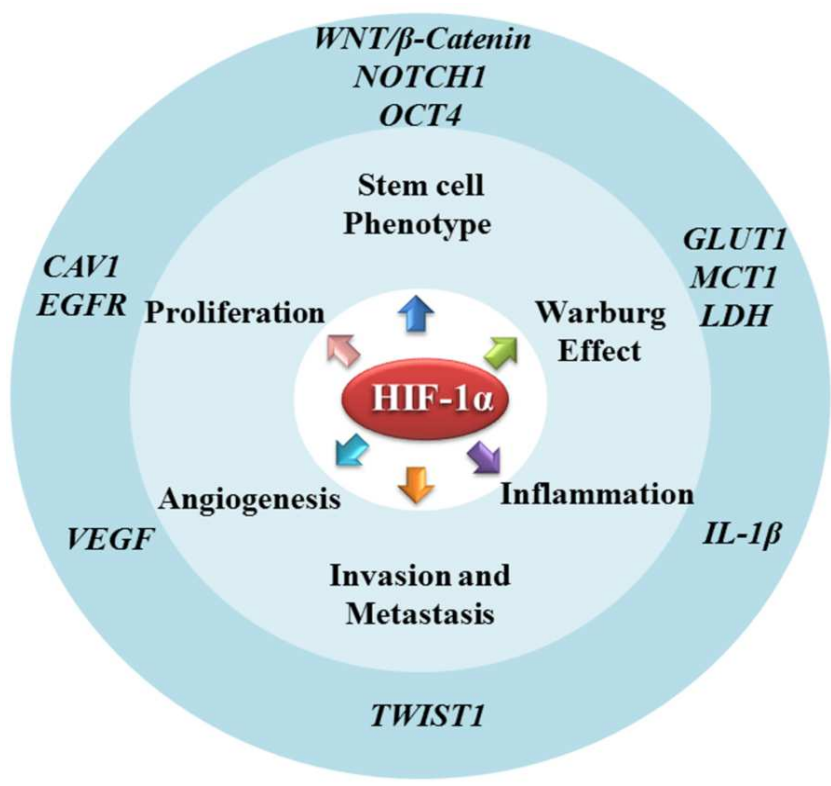

Fig. 2. HIF-1 $\alpha$ is responsible for stimulating many biological processes. In cancer, HIF-1 $\alpha$ activates the stem cell phenotype through the upregulation of OCT4, NOTCH1 and WNT/ $\beta$-catenin signalings. The activation of the metabolic enzymes Glucose Transporter (GLUT1), Monocarboxylate Trasporter (MCT1) and LDH (Lactate Dehydrogenase enzyme) by HIF-1 $\alpha$ regulates the Warburg Effect. HIF-1 $\alpha$ supports inflamation and metastasis via upregulation of Interleukin (IL)-1 $\beta$ and TWIST1, respectively. HIF- $1 \alpha$ also regulates the VEGF gene family, inducing angiogenesis in cancer. Regarding cell proliferation pathways, HIF signaling activates Caveolin 1 (CAV1), culminating in the activation of Epidermal Growth Factor Receptor (EGFR) and Extracellular-Signal-Regulated Kinases (ERK) 


\section{Hypoxia-Inducible Factor-1 and Immune Regulation}

Recently, a comprehensive study performed by Tannahill et al. (2013) directly addressed the role of HIF-1 in Interleukin (IL)-1 $\beta$ (a proinflammatory) cytokine secretion from macrophages stimulated with Lipopolysaccharide (LPS), which was derived from the external membrane of gram-negative bacteria (Yanti, 2011). HIF-1 binds to the promoter region of IL-1 $\beta$, allowing optimal transcription of pro-IL-1 $\beta$, the inactive form that generates IL- $1 \beta$ through Caspase 1 activity. Research has shown that the accumulation of succinate is essential for HIF-1 $\alpha$ stability because high levels of succinate may directly inhibit PHD. Examining this mechanism, Tannahill et al. (2013) explained how cytoplasmatic succinate increases after LPS stimulation (and the Warburg effect of the activated macrophage) and this may regulate HIF-1 $\alpha$ stability and subsequent IL- $1 \beta$ secretion. IL- $1 \beta$ is a cytokine that has been linked to cancer progression in murine models, as observed in cell lines of squamous cell carcinoma and melanoma (Chen et al., 2012; Voronov et al., 2003; Cheng et al., 2009). Regarding other proinflammatory cytokines, research has shown that IL-1 $\beta$ promotes cancer cell proliferation and is associated with cancer development during the initial stages, but it can also invoke antitumor immunity in later phases, where immune deviation is important for tumor establishment. These antagonistic effects of IL$1 \beta$ explain why opposing therapies were efficacious in murine models versus clinical trials. Administration of IL-1 $\beta$ resulted in antitumor activity in melanoma patients, whereas administration of IL-1 $\beta$ inhibitors showed promising results in cancer murine models (reviewed by Lewis et al., 2006).

In another study (Loboda et al., 2009), the authors described the inhibitory effects of HIF-1 on IL-8 expression in human endothelial cells. This result is interesting because IL-8 is known for its proangiogenic activity, which seems inconsistent because HIF- $1 \alpha$ is a hypoxia stress-responsive factor. Similar to its role in angiogenesis, IL-8 can improve cell survival, proliferation and metastasis (Waugh and Wilson, 2008). Therefore, this cytokine is considered a promising target for cancer immunotherapies. Loboda et al. (2009) also discussed the inhibitory effect of HIF-1 on Heme-Oxygenase-1 (HO-1) expression, which is an enzyme that catalyzes the degradation of heme in billiverdin, $\mathrm{CO}$ and $\mathrm{Fe}^{+2}$. HO1 is associated with cell protection against a number of oxidative agents (including heme itself); thus, the inhibitory effect of HIF-1 $\alpha$ on HO-1 seems counterintuitive as well. Additionally, two other studies (Li and Dai, 2004; Dawn and Bolli, 2005) have shown opposite results, in which HIF-1 $\alpha$ promotes HO-1 expression. Because of these opposing results, it is difficult to conclude if HIF- $1 \alpha$ expression is detrimental or beneficial to the cancer microenvironment and tumor establishment in relation to IL-8 and HO-1. HIF-1 $\alpha$ expression may be beneficial to the host by inhibiting IL-8 and HO-1, but it could be detrimental if it promotes HO-1 activity. In the latter case, it would increase cancer cell survival and potentially change the immune response indirectly. HO-1 expression by innate immune cells is linked to Treg generation, which is a lymphocyte $\mathrm{T}$ CD4+ helper subtype that is associated with cancer progression by inhibiting antitumor immunity (reviewed by Mougiakakos et al., 2010).

In addition to these controversial results, HIF- $1 \alpha$ is considered an important component of innate immune cell-mediated inflammation. In the mouse global trauma hemorrhagic shock model, partial deletion of HIF-1 was associated with reduced Tumor Necrosis Factor $\alpha(\mathrm{TNF} \alpha)$, IL-1 $\beta$, inducible Nitric Oxide Synthase (iNOS) and Cyclooxygenase 2 (COX2) (the inflammatory biomarkers; Yanti et al. 2011) levels in the ileal mucosa, which was correlated with reduced lung and intestinal injury (Feinman et al., 2010). Van Uden et al. (2008) found evidence of Nuclear Factor $(\mathrm{NF})-\kappa \mathrm{B}$ binding on the promoter region (mediating the expression) of HIF-1 $\alpha$. This study correlated two crucial transcription factors and directly linked HIF-1 $\alpha$ expression to inflammatory stimuli (Pires et al. 2013). It also provided a possible positive feedback mechanism within the inflammatory milieu because HIF-1 appears to be important for proinflammatory cytokine expression (Fig. 3).

HIF-1 can also influence the expression of NOS in macrophages that are polarized for M1 (proinflammatory), thereby supporting this phenotype (Takeda et al., 2010). Interestingly, HIF-2 $\alpha$ is expressed in macrophages polarized for the M2 phenotype and drives the expression of arginase (Takeda et al., 2010). In the tumor microenvironment, the presence of these two phenotypes is linked to opposite prognostic values, where M1 macrophages are usually associated with a good prognosis and M2 with a poor one (reviewed by Biswas et al., 2008). Recently, an interesting study from Casazza et al. (2013) showed that HIF-2, unlike HIF-1, was associated with NF- $\kappa \mathrm{B}$ induction and subsequent inhibition of Neuropilin-1 (Nrp-1)-mediated migration of macrophages. Through this mechanism, the 
macrophages were restricted to hypoxic areas and differentiated to a tumor-supportive phenotype. Both studies linked HIF-2 with tumor-supportive immune responses through directly regulating the innate response and, consequently, affecting the adaptive response as well. As such, it is tempting to speculate about therapies that inhibit the effects of HIF-2 or even induce HIF-1 $\alpha$ in Tumor-Associated Macrophages (TAMs) as a way to inhibit the M2 phenotype in these cells, at least in the tumor microenvironment. Complex studies addressing the direct role of HIF-1 in innate immune cells could add important perspectives related to this transcription factor. In this sense, HIF-1 could have antagonistic effects during different periods of cancer development. Its proinflammatory effects could be detrimental in the early phase, inducing cell proliferation by cytokine secretion; however, it could also support the M1 phenotype of TAMs, thereby inducing a protective anti-tumor immune response.

Furthermore, HIF-1 might influence on Natural Killer (NK) cells activity, another important immune cell in antitumor response (Fernandez et al., 1999). HIF-1 stabilization are associated to a lower expression of surface receptors like NKp46, NKp30, NKp44 and NKG2D in re2sponse to IL-2 and other activating cytokines (Balsamo et al., 2013). HIF-1 expression on cancer cells have been linked to MICA shedding (a component of the major histocompatibility complex family and ligand of NKG2D, an activating receptor found in NK and T CD8 cells), reducing the ability of $\mathrm{NK}$ cells to recognize malignancy. Besides that, soluble MICA reduces NKG2D surface expression on NK cells, further reducing the NK activity on tumor sites (Barsoum and Faleskog, 2011; Siemens et al., 2009).

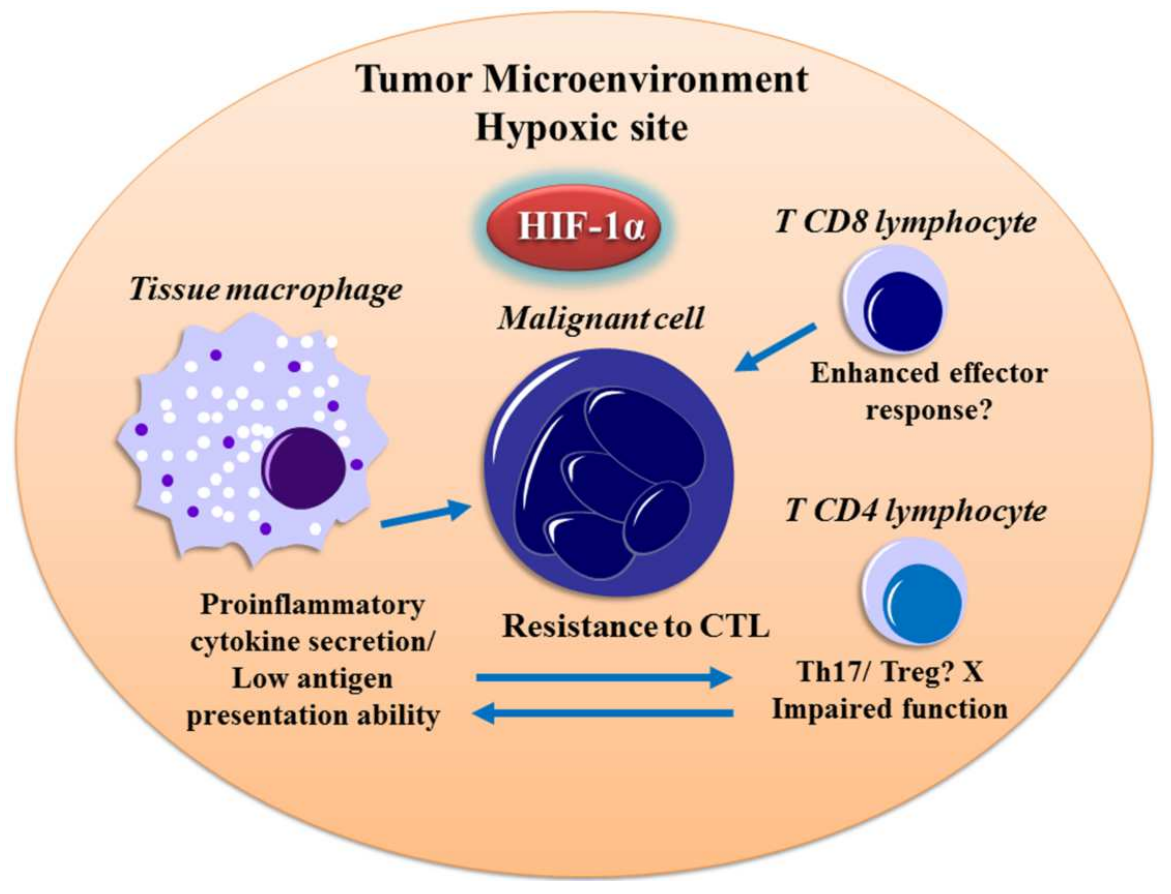

Fig. 3. HIF-1 $\alpha$ influence on immune response against cancer cells. Under hypoxic environment, both cancer and immune effector cells express HIF-1 $\alpha$ as a crucial adaptive transcription factor. This implies different kinds of interaction among cells within this microenvironment. Cancer cells seems to be more resistant to several insults, including TCD8 mediated Cytotoxic Lytic (CTL) and secrete several chemokines, like CCL28 and other soluble factors. Macrophages secrete proinflammatory cytokines, reduce antigen presentation ability and depending on HIF-1 or HIF-2 expression can drive into M1 or M2 phenotype. The lower ability of antigen presentation in this environment is another factor that might contribute to an insufficient Tcell response. Besides that, HIF-1 $\alpha$ is associated to Treg development or inhibiton, depending on the circumstances, as well as impaired T CD4 proliferation. Contradictory results about the HIF-1 $\alpha$ influence on TCD8 activity are also present. It seems that HIF-1 $\alpha$ expression supports the cancer cell development, however, as shown by different studies, HIF-1 $\alpha$ stabilization, under different circumstances, can drive macrophage M1 development, enhanced T CD8 activity and Th17 polarization, features that can drive antitumor response. Although HIF$1 \alpha$ is associated to a lower NK mediated response, the final role of each immune effector and the type of response mediated by HIF- $1 \alpha$ expression can define the tumor fate 
In the adaptive immune response, HIF-1 role is under debate. Initially, HIF-1 was associated to T cell functional inhibition (Reyren et al., 2007; Lukashev et al., 2006; Kurobe et al., 2010). In aggreement with this, Ben-Shoshan et al. (2008) demonstrated a role of HIF-1 on Treg development. However, it is important to note that HIF-1 stabilization is induced by several stimuli on $\mathrm{T}$ cells in different levels. $\mathrm{T}$ Cell Receptor (TCR) activation induces high HIF-1 activity, higher than hypoxia it self, as well as IL-6, a proinflammatory cytokine (Nakamura et al., 2005). Thus, HIF-1 activity can be modulated and its levels vary a lot on different circumstances, what may generate distinct phenotypes. HIF-1 stabilization is also related to a lower ability of $\mathrm{T}$ cells to proliferate and lower T CD8 activity (Lukashev et al., 2006), although Caldwell et al. (2001) had demonstrated different results. Furthermore, HIF-1 seems to regulate the secretion of CCL28 by cancer cells, promoting Treg migration (Facciabene et al., 2011). Although tumor associated macrophages have lower antigen presentation ability, recently HIF-1 stabilization on myeloid cells was associated to increased MHC and costimullatory molecules expression promoting $\mathrm{T}$ cell activation (Bhandari et al., 2013). In addition, HIF-1 was also associated to Th17 polarization and Treg inhibition (Dang et al., 2011) as well. It is possible that the higher expression of HIF-1 on TH17 is directly linked to IL-6 induced differentiation of these subtype. Both Th17 and Treg sub-types have been linked to a poor prognosis in different types of cancers (Martin et al., 2012; Mougiakakos et al., 2010) and more research investigating Th17 induction and Treg inhibition is needed to better define the role of HIF-1 in adaptive antitumor immunity, although TH17 antitumor response have already been described in some models (Muranski et al., 2008).

Thus, HIF-1 seems to play different roles on tumor microenvironment and its level of stabilization and expression may trigger antitumor immunity or a permissive ambient for tumor progression. The majority of studies described HIF-1 as host detrimental and its expression on cancer cells are related to increased resistance to Cytotoxic Lytic (CTL) response and tumor progression. However, a better understanding on HIF-1 induced phenotypes on immune cells and the exact influence of its expression levels and molecular interactions with other players can imply new perespectives to antitumor immunity. As such, the induction of M1 macrophage phenotype, the inhibition of Treg cell and the enhancement on TCD8 function can all support a protective antitumor immunity (Fig. 3).

\section{Anti-Hif-1a Therapies}

HIF- $1 \alpha$ and its signaling pathway are indicated as molecular targets for the new generation of drugs that are part of personalized cancer therapies. Current examples of HIF-1 $\alpha$ inhibitors include EZN-2968, an oligonucleotide antisense to the HIF-1 $\alpha$ mRNA and Tanespimicina (17AAG), which interferes with the chaperone HSP90 and destabilizes the structure of HIF-1 $\alpha$. Chetomin and its analogues influence the interaction between HIF and p300, impairing its role in transcriptional regulation. Drugs have also been developed for the major regulatory targets of HIF-1 $\alpha$ in the Warburg effect: Glufosfamide and $\alpha$-cyano-4-hydroxycinnamate, which interfere with the functions of GLUT1 and MCT1, respectively (reviewed by Wilson and Hay, 2011).

\section{Conclusion}

The hypoxia survival mechanism is essential to cell survival during the crucial stages of life development or in an anoxious microenvironment, such as that found in some tissue compartments. The effects of the hypoxiasignaling pathway are widespread when considering the complexity of the biological alterations and crosstalk with other signaling pathways. However, deregulation of this pathway could promote cellular alterations that encourage disease development, such as cancer progression and an increase in tumor cell resistance to conventional treatments. Inflammation and cancer have been intricately correlated (Miller and Shukla, 2012), due to the activation of hypoxia-related proteins that are in the inflammatory cascade. HIF- $1 \alpha$ has been included this pathway as ones of the hallmarks of cancer. The understanding of the regulatory role of HIF- $1 \alpha$ in pathological conditions has advanced, however, further studies are necessary to elucidate its value as a target for therapies.

\section{Acknowledgement}

The researchers acknowledge financial support from Instituto Nacional de Câncer José Alencar Gomes da Silva (INCA)/Ministério da Saúde (MS), Fundação Carlos Chagas Filho de Amparo à Pesquisa do Estado do Rio de Janeiro (FAPERJ), Conselho Nacional de Desenvolvimento Científico e Tecnológico $(\mathrm{CNPq})$ and Instituto Nacional de Ciência e Tecnologia para o Controle do Câncer (INCTCâncer). We also thank Camila Bandeira, M.D., for her suggestions during the writing process of this study.

\section{Author's Contributions}

All authors equally contributed in this work.

\section{Ethics}

This article is original and contains unpublished material. The corresponding author confirms that all of the other authors have read and approved the manuscript and no ethical issues involved. 


\section{References}

Adikwu, E. and N. Brambaifa, 2012. Ciprofloxacin induced chondrotoxicity and tendinopathy. Am. J. Pharmacol. Toxicol., 7: 94-100. DOI: 10.3844/ajptsp.2012.94.100

Artavanis-Tsakonas, S., M.D. Rand and R.J. Lake, 1999. Notch signaling: Cell fate control and signal integration in development. Science, 30: 284: 770-6. DOI: $10.1126 /$ science. 284.5415 .770

Baker, J., J.P. Liu, E.J. Robertson and A. Efstratiadis, 1993. Role of insulin-like growth factors in embryonic and postnatal growth. Cell, 75: 73-82. DOI: 10.1016/S0092-8674(05)80085-6

Balsamo, M., C. Manzini, G. Pietra, F. Raggi and F. Blengio et al., 2013. Hypoxia downregulates the expression of activating receptors involved in NK-cell-mediated target cell killing without affecting ADCC. Eur. J. Immunol., 43:2756-64. DOI: $10.1002 /$ eji.201343448

Barsoum, I. and J. Faleskog, 2011. Micromechanical analysis on the influence of the Lode parameter on void growth and coalescence. Int. J. Solids Structures, 48: 925-938. DOI: 10.1016/j.ijsolstr.2010.11.028

Benezra, R., R.L. Davis, D. Lockshon, D.L. Turner and H. Weintraub, 1990. The protein Id: A negative regulator of helix-loop-helix DNA binding proteins. Cell, 61: 49-59. DOI: 10.1016/0092-8674(90)90214-Y

Ben-Shoshan, J., S. Maysel-Auslender, A. Mor, G. Keren and J. George, 2008. Hypoxia controls CD4+ $\mathrm{CD} 25+$ regulatory T-cell homeostasis via hypoxiainducible factor-1alpha. Eur. J. Immunol., 38:24128. DOI: $10.1002 /$ eji.200838318

Bhandari, T., J. Olson, R.S. Johnson and V. Nizet, 2013. HIF-1a influences myeloid cell antigen presentation and response to subcutaneous OVA vaccination. J. Mol, Med., 91:1199-205. DOI: $10.1007 / \mathrm{s} 00109-013-1052-y$

Biswas, S.K., A. Sica and C.E. Lewis, 2008. Plasticity of macrophage function during tumor progression: Regulation by distinct molecular mechanisms. J. Immunol., 180: 2011-7. PMID: 18250403

Boulton, T.G. and M.H. Cobb, 1991. Identification of multiple Extracellular Signal-Regulated Kinases (ERKs) with antipeptide antibodies. Cell Regul, 2: 357-71. PMID: 1654126

Cairns, R.A., I.S. Harris and T.K. Mak, 2011. Regulation of cancer cell metabolism. Nat. Rev. Cancer, 11: 8595. DOI: $10.1038 / \mathrm{nrc} 2981$

Caldwell, C.C., H. Kojima, D. Lukashev, J. Armstrong and M. Farber et al., 2001. Differential effects of physiologically relevant hypoxic conditions on $\mathrm{T}$ lymphocyte development and effector functions. J. Immunol., 167: 6140-9. PMID: 11714773
Casazza, A., D. Laoui, M. Wenes, S. Rizzolio and N. Bassani et al., 2013. Impeding macrophage entry into hypoxic tumor areas by Sema3A/Nrp1 signaling blockade inhibits angiogenesis and restores antitumor immunity. Cancer Cell, 24: 695-709. DOI: 10.1016/j.ccr.2013.11.007

Chen, M.F., M.S. Lu, P.T. Chen, W.C. Chen and P.Y. Lin et al., 2012. Role of interleukin 1 beta in esophageal squamous cell carcinoma. J. Mol. Med., 90: 89-100. DOI: 10.1007/s00109-011-0809-4

Cheng, C.Y., H.L. Hsieh, C.C. Sun, C.C. Lin and S.F. Luo et al., 2009. IL-1 beta induces urokinase-plasminogen activator expression and cell migration through $\mathrm{PKC}$ alpha, JNK1/2 and NF-kappaB in A549 cells. J. Cell Physiol., 219: 183-93. DOI: 10.1002/jcp.21669

Dang, E.V., J. Barbi, H.Y. Yang, D. Jinasena and H. Yu et al., 2011. Control of T(H)17/T(reg) balance by hypoxia-inducible factor 1. Cell, 146: 772-84. DOI: 10.1016/j.cell.2011.07.033

Dawn, B. and R. Bolli, 2005. HO-1 induction by HIF-1: A new mechanism for delayed cardioprotection? Am. J. Physiol. Heart Circ Physiol., 289: H522-4. DOI: 10.1152/ajpheart.00274.2005

Denko, N.C., 2008. Hypoxia, HIF1 and glucose metabolism in the solid tumour. Nat. Rev. Cancer, 8: 705-713. DOI: $10.1038 /$ nrc2468

Facciabene, A., X. Peng, I.S. Hagemann, K. Balint and A. Barchetti et al., 2011. Tumour hypoxia promotes tolerance and angiogenesis via CCL28 and T(reg) cells. Nature, 475: 226-30. PMID: 21753853

Feinman, R., E.A. Deitch, A.C. Watkins, B. Abungu and I. Colorado et al., 2010. HIF-1 mediates pathogenic inflammatory responses to intestinal ischemia-reperfusion injury. Am. J. Physiol. Gastrointest Liver Physiol., 299: G833-G843. DOI: 10.1152/ajpgi.00065.2010

Fernandez, N., J. Cooper, M. Sprinks, M. AbdElrahman and D Fiszer et al., 1999. A critical review of the role of the major histocompatibility complex in fertilization, preimplantation development and fetomaternal interactions. Hum. Reprod Update, 5: 23448. PMID: 10438108

Ferrara, N., H.P. Gerber and J. LeCouter, 2003. The biology of VEGF and its receptors. Nat. Med., 9: 669-76. DOI: 10.1038/nm0603-669

Foxler, D.E., K.S. Bridge, V. James, T.M. Webb and M. Mee et al., 2012. The LIMD1 protein bridges an association between the prolyl hydroxylases and VHL to repress HIF-1 activity. Nat. Cell Biol., 14: 201-208. DOI: 10.1038/ncb2424

Hanahan, D. and R.A. Weinberg, 2011 Hallmarks of cancer: The next generation. Cell, 144: 646-74. DOI: $10.1016 /$ j.cell.2011.02.013 
Hazra, B., R. Sarkar and N. Mandal, 2012. Protection of Terminalia belerica Roxb. against iron overload induced liver toxicity: An account of its reducing and iron chelating capacity. Am. J. Pharmacol. Toxicol., 7: 109-122. DOI: 10.3844/ajptsp.2012.109.122

Kaelin, W.G. Jr., 2008. The von Hippel-Lindau tumour syuppressor protein: $\mathrm{O} 2$ sensing and cancer. Nat. Rev. Cancer, 8: 865-873. DOI: $10.1038 / \mathrm{nrc} 2502$

Kaufman, D.S., 2010. HIF hits Wnt in the stem cell niche. Nat. Cell Biol., 12: 926-927.

DOI: $10.1038 / \mathrm{ncb} 1010-926$

Krantz, S.B., 1991. Erythropoietin. Blood, 77: 419-34.

Kurobe, H., M. Urata, M. Ueno, M. Ueki and S. Ono et al., 2010. Role of hypoxia-inducible factor lalpha in $\mathrm{T}$ cells as a negative regulator in development of vascular remodeling. Arterioscler. Thromb. Vasc. Biol., 30: 210-7. DOI: 10.1161/ATVBAHA.109.192666

Lewis, A.M., S. Varghese, H. Xu and H.R. Alexander, 2006. Interleukin-1 and cancer progression: The emerging role of interleukin-1 receptor antagonist as a novel therapeutic agent in cancer treatment. J. Trans. Med., 4: 48-48. DOI: 10.1186/1479-5876-4-48

Li, Q.F. and A.G. Dai, 2004. Hypoxia inducible factor-1 alpha correlates the expression of heme oxygenase 1 gene in pulmonary arteries of rat with hypoxia-induced pulmonary hypertension. Acta Biochim. Biophys. Sin., 36: 133-40. DOI: 10.1093/abbs/36.2.133

Loboda, A., A. Stachurska, U. Florczyk, D. Rudnicka and A. Jazwa et al., 2009. HIF-1 induction attenuates Nrf2-dependent IL-8 expression in human endothelial cells. Antioxid Redox Signal, 11: 150117. DOI: 10.1089/ARS.2008.2211

Lukashev, D., B. Klebanov, H. Kojima, A. Grinberg and A. Ohta et al., 2006. Cutting edge: hypoxiainducible factor lalpha and its activation-inducible short isoform I.1 negatively regulate functions of CD4+ and CD8+ T lymphocytes. J. Immunol., 177: 4962-5. PMID: 17015677

Martin, F., L. Apetoh and F. Ghiringhelli, 2012. Controversies on the role of Th17 in cancer: A TGF- $\beta$ dependent immunosuppressive activity? Trends Mol. Med., 18: 742-9. DOI: 10.1016/j.molmed.2012.09.007

Mazumdar, J., T. O’Brien, R.S. Johnson, L.J.C. Chavez and P.S. Klein et al., 2010. $\mathrm{O}_{2}$ regulates stem cells through Wnt $/ \beta$-catenin signalling. Nat. Cell Biol., 12: 1007-1013. DOI: $10.1038 /$ ncb2 102

Meijer, T.W., J.H. Kaanders, P.N. Span and J. Bussink, 2012. Targeting hypoxia, HIF-1 and tumor glucose metabolism to improve radiotherapy efficacy. Clin Cancer Res., 18: 5585-94. DOI: 10.1158/1078-0432.CCR-12-0858

Miller, J. and A. Shukla, 2012. The role of inflammation in development and therapy of malignant mesothelioma. Am. Med. J., 3: 240-248. DOI: $10.3844 /$ amjsp.2012.240.248
Mougiakakos, D., A. Choudhury, A. Lladser, R. Kiessling and C.C. Johansson, 2010. Regulatory $\mathrm{T}$ cells in cancer. Adv. Cancer Res. DOI: 10.1016/S0065-230X(10)07003-X

Muranski, P., A. Boni, P.A. Antony, L. Cassard and K.R. Irvine et al., 2008. Tumor-specific Th17-polarized cells eradicate large established melanoma. Blood, 112 :362-73. DOI: 10.1182/blood-2007-11-120998

Nakamura, H., Y. Makino, K. Okamoto, L. Poellinger and K. Ohnuma et al., 2005. TCR engagement increases hypoxia-inducible factor-1 alpha protein synthesis via rapamycin-sensitive pathway under hypoxic conditions in human peripheral $\mathrm{T}$ cells. J. Immunol., 174: 7592-9. PMID: 15944259

Nichols, J., B. Zevnik, K. Anastassiadis, H. Niwa and D. Klewe-Nebenius et al., 1998. Formation of pluripotent stem cells in the mammalian embryo depends on the POU transcription factor OCT4. Cell, 95: 379-91. PMID: 9814708

Okamoto, T., A. Schlegel, P.E. Scherer and M.P. Lisanti, 1998. Caveolins, a family of scaffolding proteins for organizing "preassembled signaling complexes" at the plasma membrane. J. Biol. Chem., 273: 5419-22. DOI: $10.1074 /$ jbc.273.10.5419

Pires, B.R., A.L. Mencalha and E. Abdelhay, 2013. Molecular mechanisms of NF- $\kappa \mathrm{B}$ on cancer promotion and progression. SIS J.

Reyren, N., S. Reyren, A.D. Caviglia, L. Fitting Kourkoutis and G. Hammerl et al., 2007. Superconducting Interfaces Between Insulating Oxides. Science, 317: 1196-1199. DOI: 10.1126/science.1146006

Sawai, A., S. Chandarlapaty, H. Greulich, M. Gonen and Q. Ye et al., 2008. Inhibition of Hsp90 downregulates mutant Epidermal Growth Factor Receptor (EGFR) expression and sensitizes EGFR mutant tumors to paclitaxel. Cancer Res., 68: 58996. DOI: 10.1158/0008-5472.CAN-07-1570

Semenza, G.L., 1998. Hypoxia-inducible factor 1 and the molecular physiology of oxygen homeostasis. J. Lab Clin Med. DOI: 10.1016/S0022-2143(98)90091-9

Semenza, G.L., 2007. Hypoxia-Inducible Factor 1 (HIF-1) pathway. Sci. STKE. DOI: 10.1126/stke. $4072007 \mathrm{~cm} 8$

Semenza, G.L., 2010. Defining the role of hypoxiainducible factor 1 in cancer biology and therapeutics. Oncogene, 29: 625-634. DOI: 10.1038/onc.2009.441

Siemens, M.E., Q. Li, M.M. Murnane, H.C. Kapteyn and R. Yang et al., 2009. High-frequency surface acoustic wave propagation in nanostructures characterized by coherent extreme ultraviolet beams. Appl. Phys. Lett., 94: 093103-093103. DOI: 10.1063/1.3090032

Takeda, N., E.L. O'Dea, A. Doedens, J.W. Kim and A. Weidemann et al., 2010. Differential activation and antagonistic function of HIF-\{alpha\} isoforms in macrophages are essential for NO homeostasis. Genes Dev., 24: 491-501. DOI: 10.1101/gad.1881410 
Tannahill, G.M., A.M. Curtis, J. Adamik, E.M. Palsson-McDermott and A.F. McGettrick et al., 2013. Succinate is an inflammatory signal that induces IL-1 $\beta$ through HIF-1 $\alpha$. Nature, 496: 23842. DOI: $10.1038 /$ nature 11986

Van Uden, P., N.S. Kenneth and S. Rocha, 2008. Regulation of hypoxia-inducible factor-1alpha by NF-kappaB. Biochem. J., 412: 477-84. DOI: 10.1042/BJ20080476

Voronov, E., D.S. Shouval, Y. Krelin, E. Cagnano and D. Benharroch et al., 2003. IL-1 is required for tumor invasiveness and angiogenesis. Proc Natl. Acad. Sci., 100: 2645-50. DOI: 10.1073/pnas.0437939100

Wang, G.L., B. Jiang, E.A. Rue and G.L. Semenza, 1995. Hypoxia-inducible factor 1 is a basic-helixloop-helix-PAS heterodimer regulated by cellular O2 tension. Proc. Natl. Ac ad. Sci., 92: 55105514. DOI: $10.1073 /$ pnas.92.12.5510

Wang, Y., O. Roche, C. Xu, E.H. Moriyama and P. Heir et al., 2012. Hypoxia promotes ligandindependent EGF receptor signaling via hypoxiainducible factor-mediated upregulation of caveolin-1. Proc. Natl. Acad. Sci., 109: 48924897. PMID: 22411794

Waugh, D.J. and C. Wilson, 2008. The interleukin-8 pathway in cancer. Clin. Cancer Res., 14: 673541. DOI: 10.1158/1078-0432.CCR-07-4843

Wilson, W.R. and M.P. Hay, 2011. Targeting hypoxia in cancer therapy. Nat. Rev. Cancer, 11: 393-410. DOI: $10.1038 / \mathrm{nrc} 3064$
Yang, M., M. Wu, S. Chiou, P. Chen and S. Chang et al., 2008. Direct regulation of TWIST by HIF-1 $\alpha$ promotes metastasis. Nat. Cell Biol., 10: 295-305. DOI: $10.1038 / \mathrm{ncb} 1691$

Yanti, 2011. Anti-metalloproteinase-9 activities of selected Indonesian zingiberaceae rhizome extracts in lipopolysaccharide-induced human vascular endothelial cells In Vitro. Am. J. Biochem. Biotechnol., 7: 1-9. DOI: 10.3844/ajbbsp.2011.1.9

Yanti, T.E. Pramudito, N. Nuriasari and K. Juliana, 2011. Lemon pepper fruit extract (Zanthoxylum acanthopodium DC.) suppresses the expression of inflammatory mediators in lipopolysaccharide-induced macrophages in vitro. Am. J. Biochem. Biotechnol., 7: 190-195. DOI: 10.3844/ajbbsp.2011.190.195

Yi, X., Y. Liang, E. Huerta-Sanchez, X. Jin and Z.X.P. Cuo et al., 2010. Sequencing of 50 human exomes reveals adaptation to high altitude. Science, 329: 7578. DOI: $10.1126 /$ science. 1190371

Zhang, H., M. Bosch-Marce, L.A. Shimoda, Y.S. Tan and J.H. Baek et al., 2008. Mitochondrial autophagy Is an HIF-1-dependent adaptive metabolic response to hypoxia. J. Biol. Chem., 283: 10892-10903. DOI: 10.1074/jbc.M800102200 